\title{
Development and Standardization of Arabic Version of Quick Speech in Noise Test
}

\author{
Original Mostafa Ahmed Mohamed Elrifaey, Afaf Ahmad Emara, Trandil Hassan El mahallawi, \\ Article \\ Amani Mohamed El-Gharib
}

Department of Audiovestibular Medicine, Faculty of Medicine, Tanta University

\begin{abstract}
Background: Development of Arabic QuickSIN established after the need to speech in noise test using was elevated in the last decade.

Objective: The purpose of this study was to Develop and standardize Arabic QuickSIN test that measures the signal-tonoise ratio loss.

Patients and Methods: 300 Sentences have words that are not highly predictable from the surrounding context. These sentences were recorded by female talker, presented in four-talker babble in three experiments. Study sample: Fifty normal hearing subjects between the ages of 18-40 years.

Results: In the first two experiments, the level of a female talker relative to that of four-talker babble was adjusted sentence by sentence to produce $50 \%$ correct scores. In experiment III, those sentences-in-babble that produced either lack of equivalence or high across-subject variability in scores were discarded. These experiments produced 10 equivalent lists, each list consists of six sentences, with one sentence at each signal-to-noise ratio of 25, 20, 15, 10, 5, and 0 decebels. Conclusion: A single QuickSIN list takes approximately one minute to administer and provides an estimate of SNR loss.
\end{abstract}

Key Words: Quick speech in noise test (QuickSIN), signal to noise ratio loss (SNR loss), speech in noise tests.

Received: 07 July 2020, Accepted: 15 October 2021

Corresponding Author: Trandil Hassan Elmahallawy, Department of Audiovestibular Medicine, Faculty of Medicine, Tanta University, Tanta, Egypt, Tel.: 01144652753, E-mail: dr.trandil@yahoo.com

ISSN: 2090-0740, 2021

\section{INTRODUCTION}

The primary complaint of hearing-impaired persons is difficulty hearing in background noise. Measuring the signal to noise ratio loss (SNR loss) is important because speech understanding in noise cannot be reliably predicted from the puretone audiogram ${ }^{[1]}$.

Speech in noise (SIN) tests can directly address the most common complaint that patients have, which is an inability to hear well in background noise. The results of these tests may indicate quite clearly if someone needs directional microphones, stronger noise reduction programming, or extra signal processing to manage the background noise. Perhaps most importantly, it provides more precision in the way the clinicians counsel patients about realistic expectations $^{[2]}$.

There are several speech-in-noise tests that can be used clinically. Speech-in-noise tests are performed either at a fixed SNR ratio or adaptive SNR ratio. Fixed SNR ratio tests measure a percent correct at a fixed SNR ratiosthat are established by the clinician prior to the test and remain unchanged throughout. Two readily available fixed SNR ratio tests are Connected Speech Test (CST) and Speech in Noise tests (SPIN) ${ }^{[2]}$.

The Connected Speech Test (CST) is a test of the intelligibility of everyday speech. It has been developed primarily for use as a criterion measure in investigations of hearing aid benefit ${ }^{[3]}$.

The test consists of 48 passages of conversationally produced connected speech, each passage concerning a familiar topic and comprising 10 sentences. Listeners are apprised of the passage topic in advance and are required to repeat the sentences one at a time. Each passage contains 25 scoring words. The test is recorded audiovisually. Audio versions of the test have been developed for use with normal hearers (CST version 1), and for hearing-impaired listeners (CST version 2). The critical difference between two scores is estimated to be the same for both audio and audiovisual administration ${ }^{[4]}$.

SPIN test consisted of eight sets of 50 sentences recorded by a male speaker and presented in speech babble. The listener is required to repeat the last word of the sentence, a monosyllabic noun. Half of the sentences in each list are high predictability ( $\mathrm{PH})$, i.e. the last word is 
highly predictable from the semantic context, and the other half are of low predictability (PL), or contextually neutral. The same word is used once in a $\mathrm{PH}$ sentence and once in a PL sentence in an attempt to separate out the various contributions of acoustic and linguistic information. In the selection of the final words, familiarity, predictability and phonetic content were carefully considered ${ }^{[5]}$.

Adaptive SNR ratio tests measure the SNR as the intensity level of either the speech or the noise is varied. These tests can be included in the routine diagnostic hearing test battery and later compared with the patient's SNR ratio improvement with amplification. Hearing in Noise Test (HINT), Quick Speech in Noise Test, Bamford-KowalBench test (BKB-SIN) and Word in Noise test (WIN) are adaptive SNR ratio tests that can be completed quickly ${ }^{[2]}$.

Hearing in noise test (HINT) was developed by Nilsson et al. for the measurement of Reception Threshold for Sentences (RTS) in quiet and in the presence of noise. The goal of the HINT is to provide a reliable and efficient tool to estimate hearing handicap, directional hearing, and hearing aid benefit ${ }^{[6]}$.

The HINT also has a children version (HINT-C) which was developed by Nillson et al. The HINTC has 13 lists of 10 sentences. Sentences used in HINT-C are from the subset of the HINT sentences that can be repeated correctly by 6 to 12 year old children ${ }^{[7]}$.

The QuickSIN test provide a quick measure of SNR loss, can quantify the benefits of directional microphones, and help the audiologist in choosing appropriate amplification options for individuals with hearing $\operatorname{loss}^{[8]}$.

A list of six sentences with five key words per sentence is presented in four-talker babble noise. The sentences are presented at signal to noise ratios which decrease in 5-dB steps from 25 (very easy) to 0 (extremely difficult). The SNRs used are: $25,20,15,10,5$ and 0 , encompassing normal to severely impaired performance in noise ${ }^{[9]}$.

The BKB-SIN was developed to overcome the shortcoming of the English QuickSIN Test especially in young children. The sentences of QuickSIN test are also lengthy which cause difficulties in testing cochlear implant (CI) users and adults with auditory memory deficits. The BKB-SIN consist of 18 lists in which list number 1-8 has 10 sentences and from 9-18 has 8 sentences. In the QuickSIN, multi-talker babble decreases in $5 \mathrm{~dB}$ steps (from +25 to $+0 \mathrm{~dB}$ SNR). In the BKB-SIN, multitalker babble increase in $3 \mathrm{~dB}$ step so that $\mathrm{SNR}$ varies from +21 to $-6 \mathrm{~dB} \mathrm{SNR}^{[10]}$.

Words in Noise (WIN) Test was developed by Wilson to measure the ability to understand speech in multitalker babble. Monosyllabic words in WIN are adapted from the Northwestern University ${ }^{[1]}$.

Clinically available WIN materials contain two lists of 35 words (female talker). Like the QuickSIN, the WIN test also uses multi-talker babble (6 talker babble). The WIN test is typically administered under headphones. For each list in the WIN test, the noise level remains constant at 80 $\mathrm{dB}$ SPL. Intensity level of the speech material decreases in $4 \mathrm{~dB}$ step to vary SNR from +24 to $0 \mathrm{~dB}$ SNR. The WIN has a total of 7 SNR conditions and 5 monosyllabic words are presented at each SNR condition ${ }^{[12]}$.

To the best of Authors' knowledge, there are no available standardized Arabic speech in noise tests in Egypt till now except Arabic version of word in noise test, developed by Rahman $^{[12]}$ and Arabic version of hearing in noise test, developed by Essawy et al. ${ }^{[13]}$. Subsequently, the present work was aiming at development and standardization of Arabic version of QuickSIN test to measure SNR loss.

\section{PATIENTS AND METHODS}

This work was performed in Audio-vestibular unit, ENT department, Tanta University with ethical approval code No. 30538. All subjects were thoroughly counselled about the procedure, stating the values, the hazards and the aim of the study. A written consent was obtained and signed by each participant. The idea of the research was explained in details to the participants. So, the participation was voluntary. The subjects could discontinue participation at any time without penalty or loss of benefits.

\subsection{Subjects:}

This study included 50 normal hearing adults. Their age ranged from 18-40 years. Normal hearing sensitivity is defined as having pure-tone air-conduction thresholds less than or equal to $25 \mathrm{~dB} \mathrm{HL}$ at audiometric test frequencies $250 \mathrm{~Hz}$ to $8000 \mathrm{~Hz}^{[14]}$. All participating subjects had normal middle ear function with normal acoustic reflex thresholds at expected levels at frequencies from 500 to $4000 \mathrm{~Hz}$ in both ears.

\subsection{Methods:}

\subsubsection{Equipment}

Puretone audiometer: Madsen Astera which is a Type-1, two channels, and PC-based audiometer with headphones of TDH39 type. Immittancemetry: Interacoustics(AT235). Zoom H1 Handy Recorder was used for recording of sentences and babble noise.

\subsubsection{Materials:}

2.2.2.1. Quick Speech in Noise test (QuickSIN) sentences that will have the following characteristics: (1) limited contextual cues with low predictability; (2) contained five key words per sentence and were able to meet people short memory capacity; (3) avoided terminology and political terms. Recording of sentences was made by a female native speaker, with professionally trained voice. She is an Audiologist and she was understanding the purpose of the test. She was instructed to maintain clarity, pace and effort while reading the sentences. The sentences were recorded on Zoom H1 Handy Recorder and they were sampled as WAV to $24-$ bit/96KHz which used for high resolution recordings. Recordings were made 
in a double-walled sound treated room with microphone was placed perpendicular to the speaker at a distance of 1 meter. Recorded sentences were RMS Equalized.

2.2.2.2. Four Multitalker (three females and one male) babble was recorded by the same recorder. The materials used in babble were from different paragraphs from Egyptian newspaper read by the talkers, each talker read different paragraph from another at same time. Then, babble noisewas transferred to computer programs and mixed with the recorded Arabic QuickSIN sentences in a fashion that enables to direct separate inputs to the audiometer (one channel can transfer sentences material and the other channel can transfer babble noise).

\subsubsection{Procedure:}

2.2.3.1. All subjects will be subjected to: Full audiological history, otological examination and basic audiological evaluation including; puretone audiometry, speech audiometry and immittancemetry.

2.2.3.1. Standardize normative data using QuickSIN materials through three experiments:

Experiment I: Subjects: Sixteen educated adult subjects with normal hearing.Stimuli: Recorded 300 time locked sentence babble combinations. Stimulus presentation: 300 sentences were presented monaurally at $70 \mathrm{~dB}$ HL. Prior to the test session, ten sentences were administered at 70 $\mathrm{dB} H \mathrm{HL}$ in a randomized manner to familiarize the subjects with the task. The 300 sentences were then presented at -1 , +2 and $+5 \mathrm{~dB}$ SNR, in that order to each subject, each SNR presented in a separate session. Scoring: One point was given for each of five key words repeated correctly in each sentence. The SNR-50 is then calculated for each sentence using a formula based on Spearman-Kärber Equation for estimating threshold at the $50 \%$ point of the psychometric function $^{[15]}$.

Experiment II: Subjects: Eight educated normal hearing adult subjects participated in experiment II. Stimuli: A new set of recordings was made based on the
SNR-50 values from experiment I after calculation of magnitude of readjustment of each recorded sentencebabble combination to bring each sentence to an expected value of $2 \mathrm{~dB}$. Stimulus presentation: The new readjusted 300 sentences were presented monaurally to each subject at $70 \mathrm{~dB}$ HL at SNR values of $0,+5$ in two separate sessions i.e. each SNR presented to each subject in separate session. Scoring: One point was given for each of five key words repeated correctly in each sentence. The SNR-50 is then calculated for each sentence using a formula based on Spearman-Kärber Equation for estimating threshold at the $50 \%$ point of the psychometric function ${ }^{[15]}$.

Experiment III: Subjects: Twenty-six normal hearing adult subjects participated in this experiment. Stimuli: A new recordings were made based on selected sentencebabble combinations. This yielded lists of six sentences each, with one sentence at each of the following signalto-noise ratios: $25,20,15,10,5$, and $0 \mathrm{~dB}$. Stimulus presentation: The formulated lists were presented monaurally at $70 \mathrm{~dB}$ HL to 26 normal hearing subjects. Scoring: The SNR-50 is then calculated for each List using a formula based on Spearman-Kärber Equation.

\section{RESULTS}

This study included 50 normal hearing subjects. These subjects were 19 males and 31 females. The mean of age was $26.5+6.01$ years. Experiment I included 16 normal hearing subjects with a mean of age $24.5+5.3$ years. Experiment II included 8 normal hearing subjects with a mean of age $26.5+7.2$ years. Experiment III included 26 normal hearing subjects with a mean of age $27.9+5.9$ years.

\subsection{Results of Experiment I:}

1. Characteristics of each participant's response as shown in table 1 .

Table (1): Shows characteristics of each participant in experiment I.

Table 1: Characteristics of each participant's response

\begin{tabular}{lcccccc}
\hline Participant No. & Age & Sex & $\begin{array}{c}\text { Mean of SNR-50 } \\
\text { for each subject }\end{array}$ & \pm & S.D & S.E \\
\hline 1 & 22 & F & -1.03 & \pm & 1.27 & 0.073 \\
2 & 21 & F & -0.96 & \pm & 1.19 & 0.069 \\
3 & 20 & F & -0.34 & \pm & 1.44 & 0.083 \\
4 & 30 & F & -1.77 & \pm & 1.01 & 0.059 \\
5 & 21 & F & -0.60 & \pm & 1.74 & 0.100 \\
6 & 38 & F & -0.93 & \pm & 1.62 & 0.094 \\
7 & 33 & M & 0.13 & \pm & 1.67 & 0.096 \\
8 & 27 & M & -0.81 & \pm & 1.56 & 0.090 \\
9 & 27 & M & -0.70 & \pm & 1.69 & 0.098 \\
10 & 26 & M & -0.88 & \pm & 1.54 & 0.089 \\
\hline
\end{tabular}




\begin{tabular}{|c|c|c|c|c|c|c|}
\hline 11 & 20 & $\mathrm{~F}$ & -0.91 & \pm & 1.42 & 0.082 \\
\hline 12 & 19 & $\mathrm{~F}$ & -0.84 & \pm & 1.64 & 0.095 \\
\hline 13 & 21 & $\mathrm{~F}$ & -0.50 & \pm & 1.78 & 0.103 \\
\hline 14 & 22 & $\mathrm{~F}$ & -0.36 & \pm & 1.61 & 0.093 \\
\hline 15 & 22 & $\mathrm{M}$ & -0.91 & \pm & 1.57 & 0.091 \\
\hline 16 & 24 & $\mathrm{M}$ & -0.58 & \pm & 1.62 & 0.093 \\
\hline
\end{tabular}

S.E: Standard error of the mean; F: Female; M: Male.

\section{Normalization of the SNR values of the sentence- babble pairs:}

One point was given for each of five key words repeated correctly in each sentence. The SNR-50 for each sentence was calculated for using a formula based on the SpearmanKärber Equation.

\section{Standardization of sentence-babble pairs based on the normative data:}

An across-subject average SNR-50 and standard deviation (SD) were obtained for each sentence. These values were used to adjust the SNR on a sentence-bysentence basis to an expected value of $2 \mathrm{~dB}$. So, Experiment I provided, for each sentence-babble combination, SNR-50 values for each subject.

\subsection{Results of experiment II:}

The across-subject average SNR-50 for the eight normal hearing subjects was $2.09 \mathrm{~dB}$. The across-sentence average of the across-subject standard deviations was $0.53 \mathrm{~dB}$, giving a standard error of the mean for the eight normal-hearing subjects of $0.29 \mathrm{~dB}$.

1. Testing the statistical equivalence of the readjusted sentence babble combination as shown in table 2 .

Table (2): The statistical equivalence of the readjusted sentence babble combination.

2. Across sentence SNR-50 score average for each normal hearing subject as shown in table 3 .

Table (3): The across sentence SNR-50 score average for each subject participated in experiment II.

3. Testing the homogeneity of data of experiment II:Levene test was used to test the homogeneity of data of experiment II and $P$ value $=0.001$ indicating statistically significant for data homogeneity denoting good equalized sentence-babble combinations.

Table 2: Testing the equivalence of the readjusted sentence babble combination:

\begin{tabular}{lc}
\hline The across subject average of mean for 8 normal subject & 2.098 \\
\hline The across sentence average of SD of 8 normal subjects & 0.5328 \\
Standard error & 0.29 \\
$95 \% \mathrm{CI}$ & $1.94-2.26$ \\
\hline
\end{tabular}

CI: Confidence interval.

Table 3: Across sentence SNR-50 score average for each normal hearing subjects:

\begin{tabular}{lccccc}
\hline Participant No. & Age in years & Sex & Mean & \pm & S.D \\
\hline 1 & 22 & F & 2.192 & \pm & 0.711 \\
2 & 21 & F & 2.375 & \pm & 0.495 \\
3 & 20 & M & 1.722 & \pm & 1.085 \\
4 & 30 & M & 2.225 & \pm & 0.683 \\
5 & 21 & M & 2.103 & \pm & 0.806 \\
6 & 38 & F & 2.085 & \pm & 0.844 \\
7 & 33 & F & 2.080 & \pm & 0.881 \\
8 & 27 & F & 2.006 & \pm & 0.8744 \\
\hline
\end{tabular}




\section{Selection of sentences:}

Data from experiment 2 used to select sentences that were equivalent for normal hearing subjects.First step selection: Each sentence with better than average variability with $0.1 \mathrm{~dB}$; (less than average SD of the 8 normal subjects by 0.1$)$ was retained. So, this selection step eliminated 186 sentences; yielding 114 sentences. The second step selection: (a) Grand average of retained sentences from the previous step was calculated taking in account all data points. (b) Sentences with across subject average SNR50 which were within 0.43 of the grand average value were retained; i.e. each sentence with average ranging from 2.86 - 1.99 was retained. So, this selection step eliminated six sentences that were out of the 0.43 grand average; yielding 108 sentences.

5. Creation of Lists:These 108 sentences were divided randomly into 18 lists. Each list consists of six sentences, with one sentence at each of the following signal-to-noise ratios: $25,20,15,10,5$ and $0 \mathrm{~dB}$. These lists, and their sentences were chosen randomly using a computerized random sampling software.Finally, the lists were labeled from 1 to 18 .

\subsection{Results of experiment III:}

3.3.1. Assessment of the statistical equivalence of formulated lists:Each list SNR-50 score was calculated using Spearman Karber equation. Across lists average for each subjects, Standard deviation, Standard Error and Range were calculated. The across subject average for 26 normal hearing subjects was 2.5 nearly identical to Grand average of all data points of experiment II.Also for assessment of statistical equivalence for each lists, the across subjects mean of each single lists, SD, SE and 95\% confidence interval of mean were calculated. The average of across lists means was 2.58 with SD of 0.64 giving S.E. of mean 0.2 and $95 \%$ CI of mean $2.28-2.88$.

3.3.2. Descriptive data for each subject; giving mean, SD, SE, and Range as shown in table 4.

3.3.3.Assessment of equivalence for each lists, the across subjects mean of each single lists, SD, SE, and $95 \%$ confidence interval of mean were calculated. The average of across lists means was 2.58 with SD of 0.64 giving S.E. of mean 0.2 and $95 \%$ CI of mean $2.28-2.88$ (Table 5). Figure 1 shows the $95 \%$ CI of mean for each list.

Table 4: Descriptive data for each subject; giving mean, SD, SE, and Range

\begin{tabular}{|c|c|c|c|c|c|c|c|c|c|}
\hline Patient No. & Age & Sex & Mean & \pm & SD & SE & \multicolumn{3}{|c|}{ Range } \\
\hline 1 & 27 & $\mathrm{~F}$ & 2.94 & \pm & 1.474 & 0.347 & -1 & - & 4 \\
\hline 2 & 26 & M & 2.67 & \pm & 0.970 & 0.229 & 0 & - & 3 \\
\hline 3 & 20 & $\mathrm{~F}$ & 1.83 & \pm & 1.383 & 0.326 & -4 & - & 3 \\
\hline 4 & 19 & F & 2.17 & \pm & 1.505 & 0.355 & -4 & - & 3 \\
\hline 5 & 21 & M & 2.39 & \pm & 0.850 & 0.200 & -1 & - & 2 \\
\hline 6 & 22 & M & 2.67 & \pm & 0.970 & 0.229 & 0 & - & 3 \\
\hline 7 & 22 & $\mathrm{~F}$ & 2.67 & \pm & 0.970 & 0.229 & 0 & - & 3 \\
\hline 8 & 24 & M & 2.44 & \pm & 1.504 & 0.354 & -4 & - & 3 \\
\hline 9 & 32 & $\mathrm{~F}$ & 2.83 & \pm & 1.425 & 0.336 & -1 & - & 4 \\
\hline 10 & 39 & $\mathrm{~F}$ & 2.67 & \pm & 0.970 & 0.229 & 0 & - & 3 \\
\hline 11 & 23 & M & 2.44 & \pm & 1.504 & 0.354 & -4 & - & 3 \\
\hline 12 & 24 & $\mathrm{~F}$ & 2.83 & \pm & 1.425 & 0.336 & -1 & - & 4 \\
\hline 13 & 26 & M & 2.50 & \pm & 1.581 & 0.373 & -4 & - & 3 \\
\hline 14 & 29 & M & 2.94 & \pm & 1.349 & 0.318 & -1 & - & 4 \\
\hline 15 & 30 & $\mathrm{~F}$ & 2.44 & \pm & 1.504 & 0.354 & -4 & - & 3 \\
\hline 16 & 21 & $\mathrm{~F}$ & 2.67 & \pm & 0.970 & 0.229 & 0 & - & 3 \\
\hline 17 & 34 & $\mathrm{~F}$ & 2.28 & \pm & 0.826 & 0.195 & -1 & - & 2 \\
\hline 18 & 33 & $\mathrm{~F}$ & 2.67 & \pm & 0.970 & 0.229 & 0 & - & 3 \\
\hline 19 & 32 & M & 2.61 & \pm & 0.916 & 0.216 & 0 & - & 3 \\
\hline 20 & 37 & $\mathrm{~F}$ & 2.67 & \pm & 0.970 & 0.229 & 0 & - & 3 \\
\hline 21 & 22 & $\mathrm{~F}$ & 2.67 & \pm & 0.970 & 0.229 & 0 & - & 3 \\
\hline 22 & 26 & $\mathrm{M}$ & 2.56 & \pm & 1.542 & 0.364 & -4 & - & 3 \\
\hline 23 & 32 & $\mathrm{~F}$ & 2.67 & \pm & 0.970 & 0.229 & 0 & - & 3 \\
\hline 24 & 27 & $\mathrm{M}$ & 2.67 & \pm & 0.970 & 0.229 & 0 & - & 3 \\
\hline 25 & 35 & $\mathrm{~F}$ & 2.67 & \pm & 0.970 & 0.229 & 0 & - & 3 \\
\hline 26 & 40 & $\mathrm{~F}$ & 2.61 & \pm & 0.916 & 0.216 & 0 & - & 3 \\
\hline Mean & & & 2.58 & \pm & 0.46 & 0.236 & & 5 & \\
\hline
\end{tabular}


Table 5: The single list test scores means, SD, S.E., and 95\% CI interval of mean

\begin{tabular}{|c|c|c|c|c|c|c|c|}
\hline \multirow{2}{*}{$\frac{\text { List no. }}{\text { List } 1}$} & \multicolumn{3}{|c|}{ Mean +SD } & \multirow{2}{*}{$\begin{array}{c}\text { SE } \\
0.19\end{array}$} & \multicolumn{3}{|c|}{$95 \% \mathrm{CI}$ of mean } \\
\hline & 2.27 & \pm & 0.96 & & 1.88 & - & 2.65 \\
\hline List 2 & 3.65 & \pm & 0.85 & 0.17 & 3.31 & - & 3.99 \\
\hline List 3 & 2.77 & \pm & 0.82 & 0.16 & 2.43 & - & 3.09 \\
\hline List 4 & 3.12 & \pm & 0.77 & 0.15 & 2.80 & - & 3.42 \\
\hline List 5 & 2.92 & \pm & 1.32 & 0.26 & 2.39 & - & 3.45 \\
\hline List 6 & 2.54 & \pm & 1.30 & 0.26 & 2.01 & - & 3.06 \\
\hline List 7 & 3.08 & \pm & 1.98 & 0.39 & 2.28 & - & 3.87 \\
\hline List 8 & 2.50 & \pm & 0.49 & 0.09 & 1.80 & - & 2.19 \\
\hline List 9 & 2.27 & \pm & 0.45 & 0.08 & 2.08 & - & 2.45 \\
\hline List 10 & 3.62 & \pm & 1.52 & 0.29 & 2.99 & - & 4.23 \\
\hline List 11 & 1.31 & \pm & 1.80 & 0.35 & 0.057 & - & 2.03 \\
\hline List 12 & 2.19 & \pm & 0.94 & 0.18 & 1.81 & - & 2.57 \\
\hline List 13 & 2.31 & \pm & 0.93 & 0.18 & 2.30 & - & 2.77 \\
\hline List 14 & 2.54 & \pm & 0.58 & 0.11 & 2.30 & - & 2.77 \\
\hline List 15 & 2.00 & \pm & 0.00 & 0.00 & 2.00 & - & 2.00 \\
\hline List 16 & 2.62 & \pm & 0.33 & 0.063 & 1.98 & - & 2.24 \\
\hline List 17 & 2.19 & \pm & 0.40 & 0.08 & 2.03 & - & 2.35 \\
\hline List 18 & 3.62 & \pm & 0.64 & 0.16 & 3.36 & - & 3.87 \\
\hline Mean & 2.58 & + & 0.645 & 0.20 & 2.28 & - & 2.88 \\
\hline
\end{tabular}

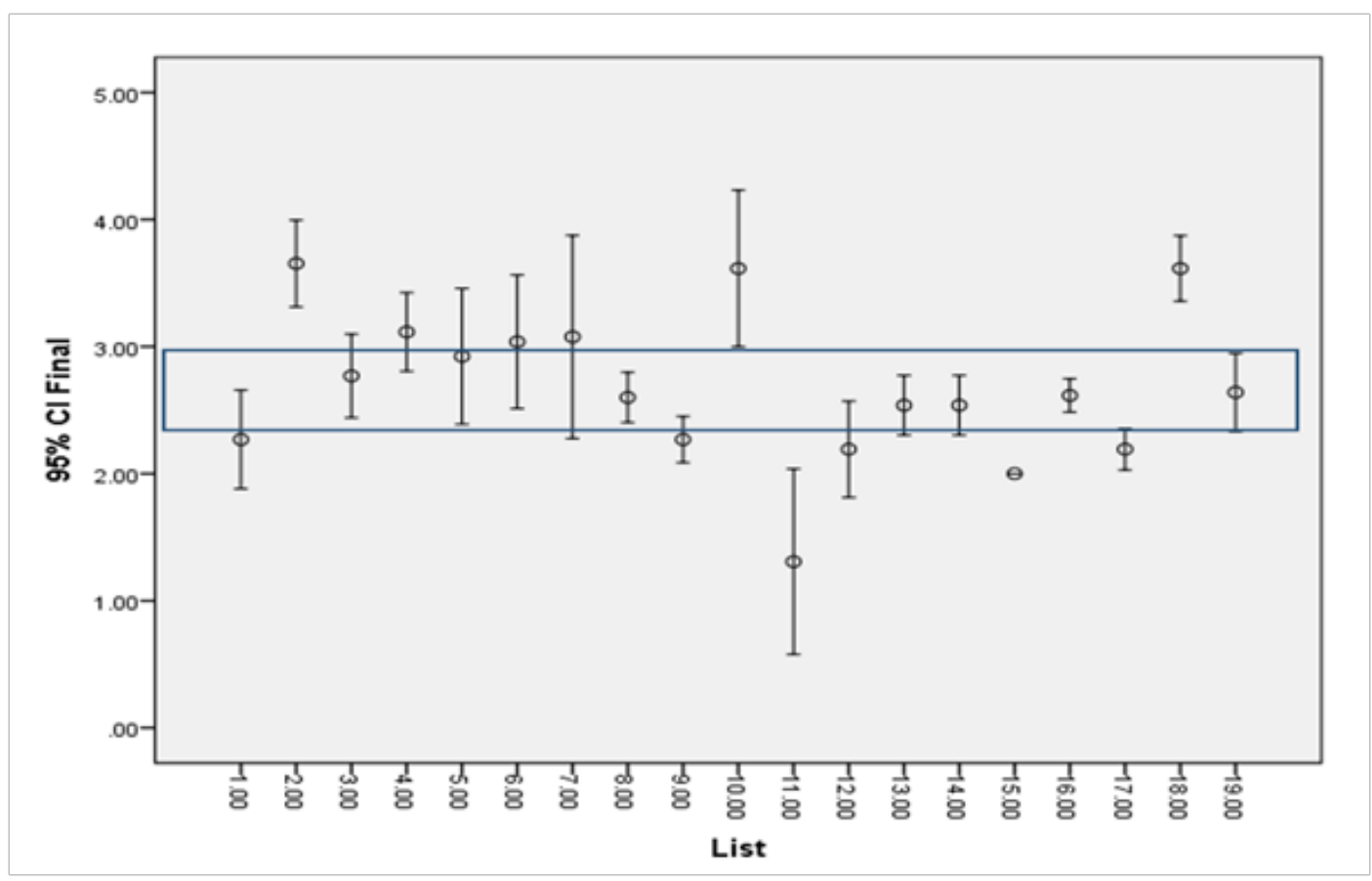

Fig. 1: The 95\% CI of mean for each list.

*No. 19 reveals to the $95 \%$ CI of mean for all lists giving 14 lists that fell in this interval. 


\subsubsection{Selection of equivalent lists:}

(i) first step of list selection: Any list with 95\% confidence interval outside the $95 \%$ confidence interval of the mean of all lists was excluded. So, four lists were excluded (2,10, 15 and 18); resulted in 14 lists. (ii) The second selection of lists:was based on the standard error of the mean of all lists i.e. any list with SE more than 0.2 was excluded. So, the final equivalent lists retained after above two selection steps were; (Lists 16,12,13,14,8,17,1,3,9 and 4). Table (6): The excluded lists from the 2 nd selection step.Table (7): The final selected lists from 2 nd selection step.

\subsubsection{Comparing one list, two lists and three lists among 26 normal hearing subjects:}

The 26 normal subjects were subjected to 6 paired lists; pairs were selected from the final equivalent 10 lists. They were also subjected to four triple list tests i.e. each subject was subjected to 3 lists. Analysis of variance (ANOVA) was done to compare the mean of the single lists, paired lists, and the triple lists across the normal hearing subjects. No statistically significant difference was present. Thus, The ANOVA test showed that using of single list is sufficient to obtain accurate SNR loss score.

\subsubsection{The reliability of final ten lists:}

Cronbach's alpha test was used to test the reliability (internal consistency) and revealed a value of 0.902 which is a good indicator of reliability of the test for repeated measurements.So, the final equivalent standardized lists which can be used to measure SNR loss are 10 lists. Single list is sufficient to obtain accurate SNR loss score. Each ear can be tested in nearly one minute; 2 minute is the total time of the test.

Table 6: The excluded lists from the 2 nd selection step

\begin{tabular}{|c|c|c|c|c|c|c|c|}
\hline \multirow{2}{*}{$\frac{\text { List no. }}{\text { List } 7}$} & \multicolumn{3}{|c|}{ Mean + SD } & \multirow{2}{*}{$\frac{\mathrm{SE}}{0.39}$} & \multicolumn{3}{|c|}{$95 \% \mathrm{CI}$ of mean } \\
\hline & 3.08 & \pm & 1.98 & & 2.28 & - & 3.87 \\
\hline List 11 & 2.00 & \pm & 1.80 & 0.35 & 0.057 & - & 2.03 \\
\hline List 5 & 2.92 & \pm & 1.32 & 0.26 & 2.39 & - & 3.45 \\
\hline List 6 & 2.54 & \pm & 1.30 & 0.26 & 2.01 & - & 3.06 \\
\hline
\end{tabular}

Table 7: The final selected lists from 2nd selection step

\begin{tabular}{|c|c|c|c|c|c|c|c|}
\hline \multirow{2}{*}{$\frac{\text { List no. }}{\text { List } 16}$} & \multicolumn{3}{|c|}{ Mean + SD } & \multirow{2}{*}{$\frac{\mathrm{SE}}{0.06}$} & \multicolumn{3}{|c|}{$95 \% \mathrm{CI}$ of mean } \\
\hline & 2.12 & \pm & 0.33 & & 1.98 & - & 2.24 \\
\hline List 12 & 2.19 & \pm & 0.94 & 0.18 & 1.81 & - & 2.57 \\
\hline List 13 & 2.31 & \pm & 0.93 & 0.18 & 2.30 & - & 2.77 \\
\hline List 14 & 2.54 & \pm & 0.58 & 0.11 & 2.30 & - & 2.77 \\
\hline List 8 & 2.60 & \pm & 0.49 & 0.09 & 1.80 & & 2.19 \\
\hline List 17 & 2.69 & \pm & 0.40 & 0.08 & 2.03 & & 2.35 \\
\hline List 1 & 2.76 & \pm & 0.96 & 0.19 & 1.88 & & 2.65 \\
\hline List 3 & 2.77 & \pm & 0.82 & 0.16 & 2.43 & & 3.09 \\
\hline List 9 & 2.77 & \pm & 0.45 & 0.08 & 2.08 & & 2.45 \\
\hline List 4 & 3.12 & \pm & 0.77 & 0.15 & 2.80 & & 3.42 \\
\hline
\end{tabular}

\section{DISCUSSION}

Experiment I provided, for each sentence-babble combination, SNR-50 values for each subject. The experimentally derived SNR-50 value for each of the time-locked sentence-babble combinations ranged from -2.5 to +5.9 $\mathrm{dB}$ across the 300 sentences which is nearly identical to
Killion et al. ${ }^{[16]}$, results $(-1.3$ to $+5.9 \mathrm{~dB})$ across his 360 sentences.

While, the across-subject average SNR-50 for the 300 sentences was $-0.75 \mathrm{~dB}$, and the standard error of the mean for the 16 subjects was $0.08 \mathrm{~dB}$ unlike the Killion et al. ${ }^{[16]}$, results whose mean was $+2.5 \mathrm{~dB}$ and $\mathrm{SE}$ 
of mean was $0.3 \mathrm{~dB}$ which is larger than our SE indicating more homogeneity of our results.

This difference in our average score $(-0.75)$ with Killion et $a l .{ }^{[16]}$, value $(+2.5)$ can be attributed to two causes:(1) the difference in language of the test; (2) in experiment I, we didn't standardize the sentence-babble combination yet which was done in the second experiment.

So, we used the SNR-50 values to readjust and standardize the sentence-babble combinations to form the new recordings of the experiment II.To standardize the sentence-babble combinations, we used the individualsentence subject-data-based SNR-50 values from experiment I. By calculation of magnitude of readjustment of each recorded sentence babble combination and to standardize each sentence to an expected value of $2 \mathrm{~dB}$. According to Bentler ${ }^{[17]}$, the average value of SNR-50 for normal hearing adults equals $2 \mathrm{~dB}$ which was calculated at $70 \mathrm{~dB}$ HL presentation level.

Experiment II demonstrated that the across-subject average SNR-50 average of the across-subject standard deviations was $0.53 \mathrm{~dB}$, giving a standard error of the mean for the eight normal-hearing subjects of $0.29 \mathrm{~dB}$. These results gave us a good indicator of equivalent standardized sentences.

The eight-subject average value of $2.09 \mathrm{~dB}$ was not significantly different than the expected value of 2 . The $95 \%$ confidence interval around the average value of $2.09 \mathrm{~dB}$ was 1.94 to $2.26 \mathrm{~dB}$. The across subject standard deviation was small, even lower than Killion et al. ${ }^{[16]}$ results $(1.6 \mathrm{~dB})$, indicating good equivalence for the readjusted sentencebabble combinations.

Also, our average value $(+2.09 \mathrm{~dB})$ is nearly identical to the average SNR-50 for normal-hearing adults at $70 \mathrm{~dB}$ HL presentation level $(+2 \mathrm{~dB})$. This is a good indicator of normalized standardized sentence-babble combinations.

We used results of Experiment II to select the most equivalent standardized sentences that had good reliability across subjects and sentences. Using two selection steps: (i) each sentence with better than average variability with $0.1 \mathrm{~dB}$; (each sentence with SD less than average SD of the 8 normal subjects by 0.1 ) was retained. So, this selection step eliminated 186 sentences; yielding 114 sentences. We used the SD as factor in selection to exclude the sentences with high statistical variability and retain only sentences with low variability.(ii) Sentences with across subject average SNR-50 which were within 0.43 of the grand average value $(2.4 \mathrm{~dB})$ were retained; i.e. each sentence with average ranging from 2.86 to $1.99 \mathrm{~dB}$ was retained. So, this selection step eliminated six sentences that were out of the 0.43 grand average; yielding 108 sentences.

These combined procedures eliminated $64 \%$ of the original 300 sentences, leaving 108 sentences meeting the criteria. While, Killion et al. ${ }^{[16]}$, results revealed elimination of $75 \%$ of the original 360 sentences, leaving 89 sentences meeting the criteria.

To create lists, these 108 sentences were divided randomly into 18 lists. Each list consists of six sentences, with one sentence at each of the following signal-tonoise ratios: $25,20,15,10,5$, and $0 \mathrm{~dB}$.These lists and their sentences were chosen randomly using computerized random sampling software.

We used this choosing technique, which not used by Killion et al. ${ }^{[16]}$, because the SDs of all retained sentences ranged from 0.35 to $0.00 \mathrm{~dB}$ indicating good equivalent standardized sentence-babble combinations.

The Experiment III provided us with 10 single lists can be used to measure SNR loss in a quick way; one minute in each ear. Eliminating four lists that fall outside the 95\% $\mathrm{CI}$ of mean. And another four lists that have SE of mean more than that of all lists $(0.2 \mathrm{~dB})$. These selection steps ensured that the retained lists have low variability with high accuracy and reliability in testing.

Finally, we tried to measure the statistical power of Arabic QuickSIN lists. In another meaning, whether single list is sufficient for accurate SNR loss measurement. Analysis of variance (ANOVA) was done to compare the mean of the single lists, paired lists, and the triple lists across the normal hearing subjects. No statistically significant difference was present. Thus, ANOVA test showed that using of single list is sufficient to obtain accurate SNR loss score.

\section{CONCLUSION}

Arabic QuickSIN can be used as a reliable and standardized test for measuring SNR loss for normal hearing subjects. The final equivalent standardized lists which can be used to measure SNR loss are 10 lists. Single list is sufficient to obtain accurate SNR loss score. Each ear can be tested in nearly one minute; two minutes is the total time of the test.

Also, we recommend application of Arabic QuickSIN on a large testing sample group of Sensorineural hearing loss subjects.And we recommend, Integration of Arabic QuickSIN test in the software of clinical audiometers especially that are sold in Middle East region.

\section{CONFLICT OF INTEREST}

There are no conflicts of interest.

\section{REFERENCES}

1. Killion M C, Niquette P A. What can the pure-tone audiogram tell us about a patient's SN loss Hear J. 2000;3:46-53.

2. Taylor B. Using speech-in-noise tests to make better 
hearing aid selection decisions. Audiology Online. Retrieved from. 2011.

3. Cox R M, Alexander G C, Gilmore C. Development of the connected speech test (CST). Ear and Hearing 1987 ; 8(5 Suppl):119S-26S

4. Cox R M, Alexander G C, Gilmore C, et al. The Connected Speech Test Version 3: Audiovisual Administration. Ear and Hearing 1989; 10(1):29-32.

5. Kalikow D N, Stevens K N, Elliott L L. Development of a test of speech intelligibility in noise using sentence materials with controlled word predictability. The Journal of the Acoustical Society of America1977; 61(5):1337-51.

6. Nilsson M, Soli S D, Sullivan J A. Development of the Hearing in Noise Test for the measurement of speech reception thresholds in quiet and in noise. The Journal of the Acoustical Society of America 1994:;95(2):1085-99.

7. Nilsson M, Soli S D, Gelnett D J. Development and norming of a hearing in noise test for children. Los Angeles: House Ear Institute. 1996.

8. Sharma S, Tripathy R, Saxena U. Critical appraisal of speech in noise tests: a systematic review and survey. International Journal of Research in Medical Sciences 2016; 19: 13-21.

9. Niquette P, Gudmundsen G, Killion M. QuickSIN Speech-in-Noise Test Version 1.3. Elk Grove Village, IL: Etymotic Research. 2001.

10. Etymotic Research. Bamford-Kowal-Bench Speechin-Noise Test (Version 1.03)-User manual; 2005. Retrieved fromhttp://www.etymotic.com/ pdf/ bkbsinuser-manual.pdf.
11. Wilson R H. Development of a speech-in-multitalkerbabble paradigm to assess word-recognition performance. Journal of the American Academy of Audiology 2003;9:453-70.

12. Rahman $\mathrm{T} T$. Development and standardization of Arabic Words in Noise test in Egyptian children. International Journal of Pediatric Otorhinolaryngology 2017;108:1-7.

13. Essawy W A, Kolkaila E K, Kabbash I A et al. Development and Standardization of Arabic Hearing in Noise test (HINT).International $\mathrm{J}$ of Otorhinolaryngology and Head and Neck Surgery 2019;5: 1501-1506.

14. American National Standards Institute [ANSI] Specification for Audiometers (ANSI S3.6-1996). New York: American National Standards Institute.1996.

15. Finney D J. Statistical method in biological assay Charles Griffin: London; 1952. Cited from (Wilson R H, McArdle R A, Smith S L. An evaluation of the BKB-SIN, HINT, QuickSIN, and WIN materials on listeners with normal hearing and listeners with hearing loss. Journal of Speech, Language, and Hearing Research 2007; 4:844-56.

16. Killion $\mathrm{M}$ C, Niquette $\mathrm{P}$ A, Gudmundsen $\mathrm{G}$ I et al. Development of a quick speech-in-noise test for measuring signal-to-noise ratio loss in normal-hearing and hearing-impaired listeners. The Journal of the Acoustical Society of America 2004; 116(4):2395-405.

17. Bentler R A. List equivalency and test-retest reliability of the speech in noise test. American Journal of Audiology 2000; 1;9(2):84-100. 